Präv Gesundheitsf 2020 · 15:371-377 https://doi.org/10.1007/s11553-020-00765-1 Eingegangen: 19. November 2019

Angenommen: 5. Februar 2020

Online publiziert: 25. Februar 2020

(C) Der/die Autor(en) 2020, korrigierte Publikation 2021

\begin{abstract}
Unsere Gesellschaft wird zunehmend älter, bunter und anzahlmäßig weniger. Ältere Beschäftigte möglichst lange und gesund in Arbeit zu halten, ist daher gesellschaftlich und wirtschaftlich von großem Interesse. Für einen gesundheitsbewussten Lebensstil ist eine realistische Einschätzung gesundheitsrelevanter Verhaltensweisen (EGVh) entscheidend. Die Identifizierung von Personengruppen mit unrealistischer EGVh kann helfen, BGFAngebote (betriebliche Gesundheitsförderung) bedarfsorientierter zu gestalten und so die Chance nachhaltiger gesundheitsrelevanter Verhaltensänderungen zu erhöhen.
\end{abstract}

\section{Hintergrund und Fragestellung}

Ältere Beschäftigte sind eine besondere Zielgruppe für die betriebliche Gesundheitsförderung (BGF, [5]). Ein Förderungspotenzial gesunder Verhaltensweisen scheint mit Blick auf die aktuelle Gesundheitslage dieser Personengruppe durchaus gegeben. In der repräsentativen Studie "Gesundheit in Deutschland aktuell“ (GEDA 2014/2015-EHIS) zeigte sich für Erwachsene zwischen 45 und 64 Jahren, dass mehr als die Hälfte (54\%) von ihnen übergewichtig ist $(\mathrm{BMI} \geq 25)$ und ein Viertel (24\%) raucht. Trotz aktuell positiver Gesundheitstrends sind mehr als drei Viertel (77 \%) dieser Altersgruppe zu wenig körperlich aktiv $[7,18,25]$. Frühere Studien zeigen, dass gesundheitsbewusstere Verhaltensweisen, wie ein ausrei-

Daniela Borchart $(\mathbb{D} \cdot$ - Hans Martin Hasselhorn - Jean-Baptist du Prel

Fachgebiet Arbeitswissenschaft, Bergische Universität Wuppertal, Wuppertal, Deutschland

\title{
Zum Gesundheitsverhalten älterer Beschäftigter - inwieweit stimmen Selbsteinschätzung und Realität überein?
}

chendes Maß an körperlicher Aktivität, ein normales Körpergewicht und Nichtrauchen, im mittleren Erwachsenenalter die Gesundheit langfristig positiv beeinflussen und das Risiko für Morbidität und Mortalität minimieren können [1,23].

Als eine zentrale Determinante gesundheitsbewussten Verhaltens wird die Gesundheitskompetenz angesehen [14, 22]. Die Selbsteinschätzung gesundheitsrelevanter Faktoren (z. B. Gesundheitszustand, Verhaltensweisen) ist dafür wesentlich [14]. Demnach verhält sich eine Person umso gesünder und passt bei Bedarf ihr Verhalten umso adäquater an, je realitätsnaher ihre Einschätzung bezüglich dieser Faktoren ist (z. B. Änderung der Ernährungsgewohnheiten bei Übergewicht; [14]). Frühere Studien zeigen, dass bestimmte Personengruppen ihr Gesundheitsverhalten (GVh) weniger zutreffend einschätzen als andere. Nach Duncan et al. [6] versuchen Übergewichtige, die sich fälschlich als normalgewichtig einschätzen, seltener Gewicht $\mathrm{zu}$ reduzieren und körperlich aktiv zu sein. Van Sluijs et al. [21] schlussfolgerten in ihrer Studie zum Zusammenhang der Einschätzung der körperlichen Aktivität (kA) und der tatsächlichen Performanz, dass Studienteilnehmer mit einem besseren Gesundheitszustand und gesundheitsbewussteren Verhaltensweisen (Nichtraucher, kein Übergewicht) ihre kA eher überschätzen. Auch zeigte sich in dieser Studie, dass ältere Befragte häufiger ihre kA überschätzen. Die Identifizierung von Risikogruppen kann für die Planung selektiver und indizierter Prä- ventionsangebote von Nutzen sein, um sie zielgruppenspezifischer $\mathrm{zu}$ gestalten und so ihren Erfolg im Sinne einer dauerhaften Verhaltensänderung zu begünstigen.

Vor diesem Hintergrund untersucht dieser Beitrag bei älteren Beschäftigten den Zusammenhang der GVh-Faktoren körperliche Aktivität, Körpergewicht und Rauchen mit der Einschätzung des eigenen GVh unter Kontrolle ausgewählter soziodemographischer Aspekte und der körperlichen Gesundheit.

\section{Datengrundlage - Studiendesign und Untersuchungsmethoden}

Analysiert wurden die Daten der lidA(leben in der Arbeit)-Kohortenstudie aus dem Jahr 2018 (dritte Erhebungswelle). Die Einschätzung des eigenen Gesundheitsverhaltens wurde zu diesem Zeitpunkt erstmalig erhoben. Die Studie untersucht prospektiv die Zusammenhänge von Arbeit, Alter, Gesundheit und Erwerbsteilhabe bei Beschäftigten der Geburtsjahrgänge 1959 und 1965. Die Teilnehmer werden in regelmäßigen Abständen (bisher 2011, 2014, 2018) befragt. Die Stichprobe ist repräsentativ für die sozialversicherungspflichtig Beschäftigten beider Alterskohorten in Deutschland [19]. Seit 2018 wird die Studie gemeinsam von der Bergischen Universität Wuppertal, der Universität Duisburg-Essen, der Heinrich-HeineUniversität Düsseldorf sowie dem Institut für angewandte Sozialwissenschaft (infas) durchgeführt. Die Konzeptua- 
Tab. 1 Deskriptive Charakteristika der

Studienteilnehmer

\begin{tabular}{|c|c|}
\hline Variable & $n(\%)$ \\
\hline \multicolumn{2}{|l|}{ Geschlecht $^{\mathrm{a}}(n=3347)$} \\
\hline Weiblich & $1833(54,8)$ \\
\hline \multicolumn{2}{|l|}{ Alter $^{\mathrm{b}}(n=3347)$} \\
\hline 1959 Geborene & $1492(44,6)$ \\
\hline \multicolumn{2}{|l|}{ Bildung ( $n=3322)$} \\
\hline Hoch & $765(23,0)$ \\
\hline Mittel & $1865(55,7)$ \\
\hline Gering & $692(20,7)$ \\
\hline \multicolumn{2}{|l|}{$\mathrm{EGVh}^{\mathrm{c}}(n=3325)$} \\
\hline $\begin{array}{l}\text { "Ich tue genug für meine } \\
\text { Gesundheit" }\end{array}$ & $2132(63,7)$ \\
\hline \multicolumn{2}{|l|}{ Körperlich aktiv ( $n=3324)$} \\
\hline 3 oder mehr Tage/Woche & $1134(34,1)$ \\
\hline 1-2 Tage/Woche & $1476(44,4)$ \\
\hline$<1$ Tag/Woche & $714(21,5)$ \\
\hline \multicolumn{2}{|l|}{$\mathrm{BMI}(n=3243)$} \\
\hline $\begin{array}{l}\text { Kein Übergewicht } \\
\quad(\mathrm{BMI}<25)\end{array}$ & $995(30,7)$ \\
\hline $\begin{array}{l}\text { Übergewicht } \\
\qquad(\text { BMI } 25<30)\end{array}$ & $1406(43,4)$ \\
\hline Adipositas (BMI $\geq 30$ ) & $842(26,0)$ \\
\hline \multicolumn{2}{|l|}{ Rauchstatus $^{\mathrm{d}}(n=3324)$} \\
\hline Raucher & $903(27,0)$ \\
\hline \multicolumn{2}{|c|}{ Körperliche Gesundheit $(n=3285)$} \\
\hline Gut & $1095(33,3)$ \\
\hline Moderat & $1096(33,4)$ \\
\hline Schlecht & $1094(33,3)$ \\
\hline \multicolumn{2}{|c|}{$\begin{array}{l}\text { EGVh Einschätzung gesundheitsrelevanter } \\
\text { Verhaltensweisen, BMI Body Mass Index } \\
\text { Referenzgruppen (nicht dargestellt): } \\
\text { aMännliches Geschlecht } \\
\text { b1965 Geborene } \\
\text { 'Personen, die angaben, eher nicht/auf } \\
\text { keinen Fall genug für ihre Gesundheit zu tun } \\
\text { dNichtraucher }\end{array}$} \\
\hline
\end{tabular}

lisierung und das Design der Studie werden ausführlich an anderer Stelle beschrieben [9, 19]. Zum Zeitpunkt der dritten Erhebungswelle (2018) waren die Teilnehmer 53 und 59 Jahre alt. In die Analyse gingen die Daten von 3347 Befragten ein, die zum Zeitpunkt der Erhebung in einem sozialversicherungspflichtigen Arbeitsverhältnis mit mindestens einer Stunde pro Woche beschäftigt waren.
Variablen

\section{Einschätzung des eigenen Gesundheitsverhaltens}

Die Befragten sollten ihr GVh anhand der Frage einschätzen: „Wenn Sie die letzten 12 Monate zusammen betrachten, wie schätzen Sie Ihr eigenes Gesundheitsverhalten (z.B. Ernährung, Sport, Fitness) ein? Stimmt die folgende Aussage: Ich tue genug für meine Gesundheit?" Die Frage beinhaltete eine vierstufige Antwortskala, die für die Analysen dichotomisiert wurde (ja, auf jeden Fall/eher ja vs. eher nein/nein, auf keinen Fall).

\section{Gesundheitsverhalten}

Analysiert wurden kA, Körpergewicht und Rauchverhalten als ausgewählte GVh-Faktoren, die in früheren Untersuchungen signifikant mit der EGVh assoziiert waren $[6,21]$. Zur Erfassung der kA sollten die Teilnehmer angeben, an wie vielen Tagen pro Woche sie sich in ihrer Freizeit für mindestens 30 Minuten körperlich so anstrengen, dass sie ins Schwitzen oder außer Atem kommen. Der Formulierungszusatz „ins Schwitzen oder außer Atem kommen“ wurde gewählt, um die Befragten bei ihrer Interpretation für eine mindestens mäßig anstrengende $\mathrm{kA}$, die mit einer Zunahme der Herz- und Atemfrequenz assoziiert ist, $\mathrm{zu}$ sensibilisieren [12, 17]. Die ursprünglich vier Antwortausprägungen wurden zu drei zusammengefasst (aktiv an $\geq 3$ Tagen/Woche, 1-2 Tagen/Woche, $<1$ Tag/Woche). Die Bewertung des Körpergewichts erfolgte mittels Body Mass Index $\left(\mathrm{BMI}, \mathrm{kg} / \mathrm{m}^{2}\right)$. In Anlehnung an die WHO-Klassifikation [24] wurden drei Gewichtsgruppen gebildet (kein Übergewicht: $\mathrm{BMI}<25$; Übergewicht: BMI 25 < 30; Adipositas: BMI $\geq 30$ ). $\mathrm{Zu}$ Rauchern wurde gezählt, wer angab, gelegentlich oder täglich zu rauchen.

\section{Kovariaten}

Kontrolliert wurde für Geschlecht, Alter, Bildung und körperliche Gesundheit (kGes). Diese Faktoren waren in früheren Studien mit der EGVh und dem GVh assoziiert [6, 7, 18, 21, 25]. Das Geschlecht wurde zu Befragungsbeginn vom Interviewer festgehalten. Die Alterskohortenzuteilung erfolgte nach Geburtsdatum.
Der Bildungsstatus (hoch, mittel, gering) wurde mittels der Angabe des höchsten erreichten schulischen und beruflichen Bildungsabschlusses gebildet [10]. Die kGes wurde mit der SF12-Skala „Physical health“ (PCS; SF-12v2 SOEP-Version, [16]) erfasst, welche den körperlichen Gesundheitszustand der letzten 4 Wochen beschreibt. Niedrige Werte bedeuten einen schlechteren Gesundheitszustand. Für die Analysen wurde die Skala anhand der Terzile in drei Gruppen klassiert (1: schlechtekGes, 2: moderatekGes, 3: gute $\mathrm{kGes}$ ).

\section{Analyse}

Vorab wurde der Zusammenhang der unabhängigen Variablen (UV) mit der EGVh einzeln anhand binär logistischer Regressionsmodelle auf Signifikanz geprüft. In multiplen logistischen Regressionsmodellen wurde der Einfluss der GVh-Faktoren auf die EGVh hierarchisch unter blockweiser Hinzunahme der Kovariaten untersucht (Modelle 1-3). Das gemeinsame Modell 3 wurde auf Interaktionen der GVh-Faktoren untereinander und mit den Kovariaten getestet. Zur Signifikanztestung wurde der Wald $\chi^{2}$-Test verwendet, zur Prüfung des Modellfits Nagelkerkes Pseudo- $\mathrm{R}^{2}$. Die statistischen Analysen wurden mit IBM SPSS Statistics Version 24 (International Business Machines Corporation, Armonk, NY, USA) durchgeführt.

\section{Fehlende Werte}

Zur EGVh lagen 3325 gültige Antworten vor $(99,3 \%, n=3347)$. Die Spanne der fehlenden Werte der GVh-Faktoren und Kovariaten reichte von 0-104. Fehlende Werte wurden durch multiple Imputation mittels der Fully-conditional-specification-Methode ersetzt [2].

\section{Ergebnisse}

\section{Stichprobenbeschreibung}

Die Mehrheit der Befragten war weiblich, 1965 geboren und hatte einen mittleren Bildungsstand. Etwa zwei Drittel gaben an, genug für die eigene Gesundheit $\mathrm{zu}$ tun $(64,1 \%)$. Übergewichtig oder adipös waren $69,3 \%(B M I \geq 25)$. Etwa ein Fünf- 
tel $(21,5 \%)$ war körperlich selten aktiv (aktiv an $<1$ Tag/Woche) und gut ein Viertel rauchte (27,0\%). - Tab. 1 veranschaulicht die deskriptiven Charakteristika der Stichprobe.

Alle untersuchten UV waren in den bivariaten Analysen signifikant mit der EGVh assoziiert (• Tab. 2).

\section{Zusammenhang der GVh-Faktoren mit der EGVh}

- Tab. 2 zeigt die multiplen Regressionsmodelle (dargestellt: Zustimmung der Befragten, genug für die eigene $\mathrm{Ge}$ sundheit zu tun). Über die Modelle 1-3 hinweg waren alle GVh-Faktoren signifikant mit der EGVh assoziiert. Demnach stimmen Raucher mit einer geringeren Chance gegenüber Nichtrauchern der Aussage $\mathrm{zu}$, genug für ihre Gesundheit $\mathrm{zu}$ tun. Je geringer die kA ist, desto geringer ist die Zustimmungschance. Je stärker das Übergewicht ist, desto geringer ist die Zustimmungschance. Unter Kontrolle der Kovariaten verringerten sich die Effekte der GVh-Faktoren auf die EGVh geringfügig.

Im multiplen Modell 1 erklärten die GVh-Faktoren 20\% der Varianz der EGVh. Die erklärte Varianz erhöhte sich unter Kontrolle der kGes in Modell 3 marginal auf $23 \%$.

\section{Zusammenhang der Kovariaten mit der EGVh}

Wie in den bivariaten Analysen waren alle Kovariaten im gemeinsamen Modell 3 mit der EGVh signifikant assoziiert. So stimmen Männer mit einer geringeren Chance als Frauen zu, genug für ihre Gesundheit zu tun und jüngere Beschäftigte mit einer geringeren Chance als die älteren. Der signifikante Bildungseffekt kehrte sich in den multiplen Modellen um. Demnach steigt mit abnehmendem Bildungsstand die Zustimmungschance. Je schlechter die kGes ist, desto geringer ist die Zustimmungschance.

Präv Gesundheitsf 2020 · 15:371-377 https://doi.org/10.1007/s11553-020-00765-1

c c Der/die Autor(en) 2020

D. Borchart · H. M. Hasselhorn - J.-B. du Prel

\section{Zum Gesundheitsverhalten älterer Beschäftigter - inwieweit stimmen Selbsteinschätzung und Realität überein?}

\section{Zusammenfassung}

Ziel der Studie. Ältere Beschäftigte sind eine besondere Zielgruppe für die Betriebliche Gesundheitsförderung. Eine realistische Einschätzung des eigenen Gesundheitsverhaltens (EGVh) ist wesentlich für die adäquate Anpassung des Gesundheitsverhaltens (GVh) bei Bedarf. Die EGVh kann nach Sozial- und Gesundheitsaspekten variieren. Daher wird untersucht, wie GVh-Faktoren auf die EGVh älterer Beschäftigter nach soziodemografischen Aspekten und der körperlichen Gesundheit (kGes) wirken. Methodik. Analysiert wurden die Daten von 3347 älteren Beschäftigten der repräsentativen lidA-Studie 2018. Die EGVh wurde mit der Aussage „Ich tue genug für meine Gesundheit" erfasst. Als GVh-Faktoren wurden die körperliche Aktivität (kA), der BMI und das Rauchverhalten untersucht. Mit hierarchischen logistischen Regressionen wurde der Einfluss der GVh-Faktoren auf die EGVh kontrolliert für Geschlecht, Alter, Bildung und kGes analysiert. Um das Zusammenspiel der unabhängigen Variablen hinsichtlich der EGVh genauer zu untersuchen, wurde zudem auf Interaktionen getestet.
Ergebnisse. Gesündere Verhaltensweisen sind positiv mit der Zustimmung, genug für die eigene Gesundheit zu tun assoziiert. Allerdings zeigten die gefundenen Interaktionen für bestimmte Personengruppen abweichende Ergebnisse. So stimmen übergewichtige Raucher gegenüber normalgewichtigen mit einer höheren Chance der Aussage zu, genug für die eigene Gesundheit zu tun. Bei Nichtaktiven stieg die Zustimmungschance mit abnehmendem Bildungsniveau.

Schlussfolgerung. Die Identifizierung von Personengruppen, die ihre GVh weniger zutreffend einschätzen als andere, kann helfen, das Angebot an Präventionsmaßnahmen bedürfnisspezifischer zu gestalten. Das Zusammenspiel der Einflussfaktoren auf die EGVh gilt es zukünftig noch genauer zu untersuchen.

\section{Schlüsselwörter}

Bewertung des Gesundheitsverhaltens . Körperliche Aktivität · Body Mass Index . Rauchen · Mittleres Erwachsenenalter

\section{Older workers' health behavior - to what extent does self- estimation equal reality?}

\section{Abstract}

Aim. Older workers are a special target group for workplace health promotion programs. A realistic estimation of one's own health behavior (EHB) is essential to adjust health behavior adequately, if needed. EHB may vary depending on social and health aspects. Therefore, this study investigates the impact of HB-factors on EHB of older workers, considering sociodemographic aspects and physical health status (PHS).

Method. Data of 3347 older employees participating in the representative lidA-study 2018 were analyzed. EHB was measured by the agreement to the statement "My health behavior is sufficient". Examined HBfactors were physical activity (PA), BMI and smoking. Associations between HB-factors and EHB were analyzed with hierarchical logistic regression models adjusted for sex, age, education and PHS. The interplay of determinants on EHB was additionally tested with interaction terms.
Results. More favorable health patterns are associated with the agreement to the statement that HB is sufficient. However, the interaction analysis showed different linkages to EHB for some individual groups. Therefore, overweight smokers are more likely to rate their HB as sufficient than non-overweight. With non-active participants, the chance of rating $\mathrm{HB}$ as sufficient increased with lower educational levels.

Conclusions. The identification of groups with a higher chance of misperceiving HB might help to establish preventive measures more specific to their needs. Further research is needed to disentangle the complex interplay of the determinants on EHB.

Keywords

Perception of health behavior - Physical activity · Body Mass Index · Smoking · Middle aged 


\section{Originalarbeit}

\begin{tabular}{|c|c|c|c|c|}
\hline Variable & $\begin{array}{l}\text { Bivariate Analysen } \\
\text { OR (95\%-KI) }\end{array}$ & $\begin{array}{l}\text { Modell } 1\left(R^{2}=0,20\right) \\
\text { OR }(95 \%-K I)\end{array}$ & $\begin{array}{l}\text { Modell } 2\left(R^{2}=0,20\right) \\
\text { OR }(95 \%-K I)\end{array}$ & $\begin{array}{l}\text { Modell } 3\left(R^{2}=0,23\right) \\
\text { OR }(95 \%-K I)\end{array}$ \\
\hline \multicolumn{5}{|l|}{ Körperliche Aktivität ${ }^{\mathrm{a}}$} \\
\hline 1-2 Tage/Woche & $0,38(0,31-0,45)^{* * *}$ & $0,39(0,32-0,47)^{* * *}$ & $0,39(0,320,47)^{* * * *}$ & $0,39(0,32-0,47)^{* * *}$ \\
\hline$<1$ Tag/Woche & $0,17(0,14-0,21)^{* * *}$ & $0,18(0,15-0,23)^{* * *}$ & $0,18(0,15-0,23)^{* * *}$ & $0,19(0,15-0,24)^{* * *}$ \\
\hline \multicolumn{5}{|l|}{$\mathrm{BMI}^{\mathrm{b}}$} \\
\hline $\begin{array}{l}\text { Übergewicht } \\
\quad(\text { BMI } 25<30)\end{array}$ & $0,68(0,56-0,81)^{* * *}$ & $0,66(0,55-0,80)^{* * *}$ & $0,68(0,56-0,82)^{* * * *}$ & $0,70(0,57-0,85)^{* * *}$ \\
\hline Adipositas (BMI $\geq 30$ ) & $0,29(0,24-0,36)^{* * *}$ & $0,28(0,23-0,35)^{* * *}$ & $0,28(0,23-0,35)^{* * * *}$ & $0,33(0,26-0,40)^{* * *}$ \\
\hline Raucher $^{c}$ & $0,50(0,43-0,59)^{* * *}$ & $0,47(0,40-0,56)^{* * *}$ & $0,47(0,39-0,55)^{* * *}$ & $0,48(0,41-0,58)^{* * *}$ \\
\hline Männer ${ }^{d}$ & $0,77(0,67-0,89)^{* * *}$ & & $0,87(0,74-1,02)$ & $0,82(0,70-0,96)^{*}$ \\
\hline 1965 Geborene $^{e}$ & $0,84(0,73-0,97)^{*}$ & & $0,84(0,72-0,98)^{*}$ & $0,77(0,65-0,90)^{* *}$ \\
\hline \multicolumn{5}{|l|}{ Bildung $^{f}$} \\
\hline Mittel & $0,88(0,74-1,06)$ & & $1,07(0,88-1,30)$ & $1,23(1,01-1,51)^{*}$ \\
\hline Gering & $0,76(0,61-0,94)^{*}$ & & $1,13(0,89-1,43)$ & $1,39(1,08-1,77)^{* *}$ \\
\hline \multicolumn{5}{|l|}{ Körperliche Gesundheit ${ }^{g}$} \\
\hline Moderat & $0,47(0,39-0,57)^{* * *}$ & & & $0,52(0,42-0,64)^{* * *}$ \\
\hline Schlecht & $0,35(0,29-0,41)^{* * *}$ & & & $0,40(0,33-0,50)^{* * *}$ \\
\hline \multicolumn{5}{|c|}{ 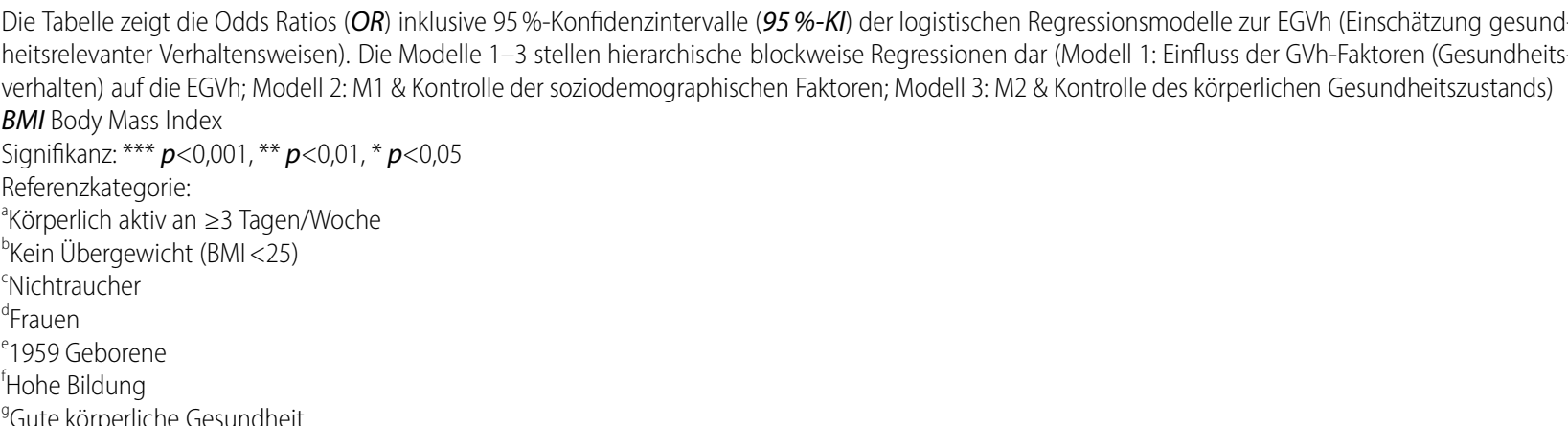 } \\
\hline
\end{tabular}

\begin{tabular}{|c|c|c|c|c|c|c|}
\hline Variable & $\begin{array}{l}\text { Modell } 3 \\
\left(R^{2}=0,23\right) \\
\text { OR }(95 \%-K I)\end{array}$ & $\begin{array}{l}\text { kA } \times \text { Geburtsjahr } \\
\left(R^{2}=0,23\right) \\
\text { OR }(95 \%-K I)\end{array}$ & $\begin{array}{l}\text { kA } \times \text { Bildung } \\
\left(R^{2}=0,23\right) \\
O R(95 \%-K I)\end{array}$ & $\begin{array}{l}\text { kA } \times \text { kGes } \\
\left(R^{2}=0,24\right) \\
\text { OR }(95 \%-K I)\end{array}$ & $\begin{array}{l}\text { Rauchen } \times \text { kGes } \\
\left(R^{2}=0,23\right) \\
\text { OR }(95 \%-K I)\end{array}$ & $\begin{array}{l}\text { Rauchen } \times \text { BMI } \\
\left(R^{2}=0,24\right) \\
\text { OR }(95 \%-K I)\end{array}$ \\
\hline \multicolumn{7}{|c|}{ Körperliche Aktivität (kA) ${ }^{\mathrm{a}}$} \\
\hline $\begin{array}{l}\text { 1-2 Tage/ } \\
\text { Woche }\end{array}$ & $0,39(0,32-0,47)^{* * *}$ & $0,38(0,29-0,51)^{* * * *}$ & $0,29(0,19-0,44)^{* * * *}$ & $0,25(0,17-0,38)^{* * * *}$ & $0,39(0,32-0,47)^{* * * *}$ & $0,39(0,32-0,47)^{* * * *}$ \\
\hline$<1$ Tag/Woche & $0,19(0,15-0,24)^{* * * *}$ & $0,26(0,19-0,36)^{* * *}$ & $0,10(0,06-0,16)^{* * *}$ & $0,08(0,05-0,13)^{* * *}$ & $0,19(0,15-0,24)^{* * * *}$ & $0,19(0,15-0,34)^{* * *}$ \\
\hline \multicolumn{7}{|l|}{$\mathrm{BMI}^{\mathrm{b}}$} \\
\hline $\begin{array}{l}\text { Übergewicht } \\
\quad(\text { BMI } 25<30)\end{array}$ & $0,70(0,57-0,85)^{* * *}$ & $0,70(0,57-0,86)^{* * * *}$ & $0,69(0,56-0,84)^{* * *}$ & $0,70(0,57-0,86)^{* *}$ & $0,69(0,57-0,85)^{* * *}$ & $0,54(0,42-0,70)^{* * *}$ \\
\hline $\begin{array}{l}\text { Adipositas } \\
\qquad(\mathrm{BMI} \geq 30)\end{array}$ & $0,33(0,26-0,40)^{* * * *}$ & $0,32(0,26-0,40)^{* * * *}$ & $0,32(0,26-0,40)^{* * *}$ & $0,32(0,26-0,40)^{* * *}$ & $0,32(0,26-0,40)^{* * *}$ & $0,24(0,19-0,31)^{* * * *}$ \\
\hline Raucher ${ }^{c}$ & $0,48(0,41-0,58)^{* * *}$ & $0,48(0,41-0,58)^{* * *}$ & $0,48(0,41-0,57)^{* * *}$ & $0,49(0,41-0,58)^{* * *}$ & $0,64(0,46-0,90)^{*}$ & $0,28(0,20-0,39)^{* * *}$ \\
\hline Männer $^{d}$ & $0,82(0,70-0,96)^{*}$ & $0,81(0,69-0,96)^{*}$ & $0,82(0,69-0,96)^{*}$ & $0,81(0,69-0,95)^{*}$ & $0,82(0,70-0,97)^{*}$ & $0,82(0,70-0,97)^{*}$ \\
\hline 1965 Geborene $^{e}$ & $0,77(0,65-0,90)^{* *}$ & $0,87(0,64-1,19)$ & $0,77(0,66-0,90)^{* *}$ & $0,77(0,66-0,91)^{* *}$ & $0,77(0,65-0,90)^{* *}$ & $0,76(0,65-0,89)^{* *}$ \\
\hline \multicolumn{7}{|l|}{ Bildung $^{f}$} \\
\hline Mittel & $1,23(1,01-1,51)^{*}$ & $1,22(1,00-1,49)$ & $0,92(0,61-1,39)$ & $1,23(1,00-1,50)^{*}$ & $1,24(1,01-1,51)^{*}$ & $1,26(1,03-1,54)^{*}$ \\
\hline Gering & $1,39(1,08-1,77)^{* *}$ & $1,37(1,07-1,76)^{*}$ & $0,78(0,49-1,26)$ & $1,38(1,08-1,77)^{*}$ & $1,40(1,09-1,79)^{* *}$ & $1,43(1,11-1,83)^{* *}$ \\
\hline \multicolumn{7}{|l|}{$\mathrm{kGes}^{\mathrm{g}}$} \\
\hline Moderat & $0,52(0,42-0,64)^{* * *}$ & $0,52(0,42-0,65)^{* * *}$ & $0,52(0,43-0,64)^{* * *}$ & $0,34(0,21-0,53)^{* * *}$ & $0,61(0,48-0,78)^{* * *}$ & $0,52(0,43-0,64)^{* * * *}$ \\
\hline Schlecht & $0,40(0,33-0,50)^{* * *}$ & $0,40(0,32-0,49)^{* * *}$ & $0,40(0,33-0,49)^{* * *}$ & $0,20(0,13-0,31)^{* * *}$ & $0,43(0,33-0,54)^{* * *}$ & $0,41(0,33-0,50)^{* * *}$ \\
\hline
\end{tabular}




\begin{tabular}{|c|c|c|c|c|c|c|}
\hline Variable & $\begin{array}{l}\text { Modell } 3 \\
\left(R^{2}=0,23\right) \\
\text { OR }(95 \%-K I)\end{array}$ & $\begin{array}{l}\text { kA } \times \text { Geburtsjahr } \\
\left(R^{2}=0,23\right) \\
\text { OR }(95 \%-K I)\end{array}$ & $\begin{array}{l}k A \times \text { Bildung } \\
\left(R^{2}=0,23\right) \\
\text { OR }(95 \%-K I)\end{array}$ & $\begin{array}{l}k A \times k G e s \\
\left(R^{2}=0,24\right) \\
\text { OR }(95 \%-K I)\end{array}$ & $\begin{array}{l}\text { Rauchen } \times \text { kGes } \\
\left(R^{2}=0,23\right) \\
\text { OR }(95 \%-K I)\end{array}$ & $\begin{array}{l}\text { Rauchen } \times \text { BMI } \\
\left(R^{2}=0,24\right) \\
\text { OR }(95 \%-K I)\end{array}$ \\
\hline $\begin{array}{l}\text { kA (<1 Tag/ } \\
\text { Woche) } \times 1965 \\
\text { Geborene }\end{array}$ & & $0,56(0,36-0,88)^{*}$ & & & & \\
\hline $\begin{array}{l}\mathrm{kA}(<1 \mathrm{Tag} / \\
\text { Woche }) \times \text { Bildung } \\
\text { mittel }\end{array}$ & & & $1,87(1,04-3,37)^{*}$ & & & \\
\hline $\begin{array}{l}\mathrm{kA}(<1 \mathrm{Tag} / \\
\text { Woche }) \times \text { Bildung } \\
\text { gering }\end{array}$ & & & $3,35(1,71-6,58)^{* * * *}$ & & & \\
\hline $\begin{array}{l}\text { kA ( } 1-2 \text { Tage/ } \\
\text { Woche) } \times \text { kGes } \\
\text { schlecht }\end{array}$ & & & & $1,95(0,17-3,25)^{*}$ & & \\
\hline $\begin{array}{l}\text { kA }(<1 \mathrm{Tag} / \\
\text { Woche }) \times \text { kGes } \\
\text { moderat }\end{array}$ & & & & $2,29(1,25-4,20)^{* *}$ & & \\
\hline $\begin{array}{l}\text { kA }(<1 \mathrm{Tag} / \\
\text { Woche }) \times \text { kGes } \\
\text { schlecht }\end{array}$ & & & & $3,98(2,22-7,13)^{* * *}$ & & \\
\hline $\begin{array}{l}\text { Rauchen } \times \mathrm{kGes} \\
\text { moderat }\end{array}$ & & & & & $0,57(0,36-0,88)^{*}$ & \\
\hline $\begin{array}{l}\text { Rauchen } \times \\
\text { Übergewicht } \\
(\text { BMI } 25<30)\end{array}$ & & & & & & $1,95(1,29-2,94)^{* *}$ \\
\hline $\begin{array}{l}\text { Rauchen } \times \\
\text { Adipositas } \\
(\mathrm{BMI} \geq 30)\end{array}$ & & & & & & $2,54(1,59-4,05)^{* * *}$ \\
\hline \multicolumn{7}{|c|}{$\begin{array}{l}\text { Die Tabelle zeigt die Odds Ratios (OR) inklusive } 9 \\
\text { mit signifikanten Interaktionstermen } \\
\text { BMI Body Mass Index, } k G e s \text { körperliche Gesundh } \\
\text { Signifikanz: }{ }^{* *} p<0,001,{ }^{* *} p<0,01,{ }^{*} p<0,05 \\
\text { Referenzkategorie: } \\
{ }^{2} \text { Körperlich aktiv an } \geq 3 \text { Tagen/Woche } \\
\left.{ }^{6} \text { Kein Übergewicht (BMl }<25\right) \\
{ }^{C} \text { Nichtraucher } \\
{ }^{d} \text { Frauen } \\
{ }^{\text {}} 1959 \text { Geborene } \\
{ }^{\circ} \text { Hohe Bildung } \\
{ }^{9} \text { Gute körperliche Gesundheit }\end{array}$} \\
\hline
\end{tabular}

\section{Zusammenspiel der Einfluss- faktoren - signifikante Interaktionen}

- Tab. 3 stellt die Modelle mit den signifikanten Interaktionstermen dar. Für Raucher zeigte sich: Je stärker das Übergewicht, desto höher die Zustimmungschance. Für Nichtaktive (aktiv $<1$ Tag/ Woche) zeigte sich, dass jüngere Beschäftigte mit einer geringeren Chance als Ältere zustimmen, genug zu tun. Der Haupteffekt von Alter auf die EGVh war unter Hinzunahme der Interaktion $\mathrm{kA}^{\star}$ Alter nicht mehr signifikant. Auch zeigte sich für Nichtaktive, dass die $\mathrm{Zu}$ - stimmungschance mit abnehmendem Bildungsstand steigt. Unter Hinzunahme dieser Interaktion kehrte sich der Haupteffekt von Bildung um. Wie in der bivariaten Analyse war ein höheres Bildungsniveau mit einer höheren $\mathrm{Zu}$ stimmungschance assoziiert. Um zu klären, ob die Umkehr des Bildungseffekts auf die kA zurückzuführen ist, wurde zusätzlich der Einfluss von Bildung auf EGVh unter Kontrolle jeweils eines GVhFaktors geprüft (Modelle nicht dargestellt). In diesen Modellen zeigte sich für keine der Bedingungen eine Umkehr des Bildungseffekts. Zudem zeigte sich für
Nichtaktive, dass mit schlechterer kGes die Zustimmungschance steigt.

Die erklärte Varianz des gemeinsamen Modells 3 zeigte unter Hinzunahme der Interaktionen keine Veränderung.

\section{Diskussion}

Diese Studie untersuchte, inwieweit GVh-Faktoren mit der EGVh bei älteren Beschäftigten assoziiert sind. Dazu wurde in hierarchischen logistischen Regressionsmodellen der Zusammenhang der Faktoren kA, BMI und Rauchen mit der EGVh kontrolliert für Geschlecht, Alter, Bildung und kGes multivariat 
analysiert. Wie erwartet, gaben Beschäftigte mit gesünderen Verhaltensweisen (körperlich aktiv, kein Übergewicht und Nichtraucher) eher an, genug für die eigene Gesundheit zu tun. Für die Kovariaten zeigte sich konform mit den Ergebnissen anderer Studien $[6,21]$, dass Frauen, Ältere und Personen mit guter kGes eher zustimmen, genug zu tun.

Bei allen erwartungstreuen protektiven Haupteffekten der GVh-Faktoren auf die EGVh zeigten sich wie in früheren Studien $[6,21]$ für bestimmte Personengruppen abweichende Ergebnisse. So stimmen Nichtaktive mit schlechter kGes eher zu, genug zu tun, als Nichtaktive mit besserer kGes. Auch ist die Zustimmungschance von übergewichtigen Rauchern höher als von denen ohne Übergewicht. Möglich ist, dass bei diesen Personengruppen die individuelle Umsetzbarkeit eines gesundheitsbewussten Lebensstils die EGVh beeinflusst hat. So kann es sein, dass Personen mit schlechter kGes im Rahmen ihrer wahrgenommenen Möglichkeiten aktiv sind und dies ihre EGVh beeinflusst. Hierfür spricht u.a., dass bei körperlichen Beschwerden, wie Rückenschmerzen, Personen häufig ihre $\mathrm{kA}$ einschränken, obwohl dies zur Aufrechterhaltung und Verschlimmerung der Beschwerden beitragen kann [11]. Bei Übergewichtigen kann die Erwartung einer Gewichtszunahme bei Rauchstopp Grund dafür sein, nicht mit dem Rauchen aufzuhören $[4,8]$. Insgesamt deuten die festgestellten Interaktionen auf ein komplexes Zusammenwirken der Faktoren im Einfluss auf die EGVh hin. Unter anderem kehrte sich der in der bivariaten Analyse protektive Effekt von Bildung auf die EGVh unter Kontrolle der weiteren UV um. Demnach hätten geringer Gebildete, wenn sie sich nicht bezüglich der kontrollierten Kovariaten von höher Gebildeten unterscheiden würden, eine höhere Zustimmungschance als Hochgebildete. Bei der Testung auf Interaktionseffekte war die Interaktion $\mathrm{kA}^{\star}$ Bildung signifikant. Für Nichtaktive zeigte sich: Je geringer die Bildung, desto höher die Zustimmungschance. Der Haupteffekt von Bildung auf die EGVh war unter Hinzunahme dieser Interak- tion im Modell nicht mehr signifikant. Ob die Umkehr des Bildungseffekts allein auf die kA zurückzuführen ist, lässt sich anhand der vorliegenden Analysen nicht abschließend beurteilen. Vielmehr scheint das Zusammenspiel der verschiedenen Faktoren von Bedeutung zu sein. Dennoch stimmen die gefundenen signifikanten Ergebnisse mit denen anderer Untersuchungen überein, die für Gesundheitskompetenz und gesundheitsbezogene Verhaltensweisen einen sozialen Gradienten aufzeigen $[3,7,18$, 20, 25].

Eine Stärke dieser Untersuchung ist die Stichprobengröße. Dies ermöglichte die Testung von Unterschieden in Subgruppen (z. B. geringe kA und schlechte kGes). Die untersuchte Stichprobe ist zudem repräsentativ für die sozialversicherungspflichtig Beschäftigten der Geburtsjahrgänge 1965 und 1959 hierzulande [19]. Durch die Befragung von zwei Alterskohorten ist die Generalisierbarkeit der Ergebnisse für die gesamte Gruppe älterer Beschäftigter nur bedingt möglich. Aufgrund des querschnittlichen Untersuchungsdesigns sind richtungsweisende Aussagen über die Assoziationen der Faktoren zueinander vorsichtig zu betrachten. Ferner lässt das querschnittliche Design vorliegender Untersuchung eine klare Differenzierung zwischen einem Alters- und Kohorteneffekt für die Interpretation des signifikanten Einflusses des Alters auf die EGVh nicht zu. Die kGes wurde anhand des Gesundheitszustands der letzten vier Wochen für die Analysen herangezogen, wohingegen das Gesundheitsverhalten der letzten 12 Monate bewertet werden sollte. Eine Veränderung derkGes über diesen Zeitraum kann nicht ausgeschlossen werden. Die Aussagekraft der Ergebnisse kann durch die Parametrisierung der GVh-Faktoren eingeschränkt sein, da diese erfragt und nicht objektiv gemessen wurden. Eine Verzerrung aufgrund sozialer Erwünschtheit bei den GVh-Faktoren und der EVGh kann nicht ausgeschlossen werden. Bei der Interpretation der Ergebnisse zur kA ist auch zu berücksichtigen, dass aufgrund der Parametrisierung (Häufigkeit in Tagen pro Woche) das Aktivitätsniveau nur annäherungsweise abgeschätzt werden kann. Allerdings zeigte sich, dass die Prävalenzen der GVh-Faktoren unserer Befragten weitestgehend vergleichbar zu denen anderer repräsentativer Studien in Deutschland für die Erwachsenenbevölkerung mittleren Alters sind (GEDA 2014/2015 [7, 18, 25]; DEGS1 [12, 15]).

\section{Schlussfolgerungen und Ausblick}

Die Selbsteinschätzung gesundheitsrelevanter Faktoren gilt als wesentlicher Bestandteil der Gesundheitskompetenz [14]. Da das Morbiditäts- und Mortalitätsrisiko mit dem kumulativen Auftreten gesundheitlich riskanter Verhaltensweisen assoziiert ist [1, 23], scheint die Identifizierung von Risikogruppen, deren EGVh weniger realistisch als bei anderen ist, wichtig. Auch kann die Identifizierung von Risikogruppen helfen, die Gesundheitsberatung und das Angebot selektiver und indizierter Präventionsmaßnahmen inhaltlich zielgerichteter auf die anzusprechende Klientel auszurichten.

Dass bei älteren Beschäftigten ein Förderungspotenzial bezüglich gesunden Verhaltens besteht, bestätigen auch unsere Ergebnisse. Vergleichbar mit den Ergebnissen anderer repräsentativer Studien zum GVh von Personen im mittleren Erwachsenenalter zeigte sich für die in diesem Beitrag befragten Alterskohorten, dass die Mehrheit von ihnen übergewichtig ist, sich nicht ausreichend bewegt und etwa ein Drittel raucht [7, $12,15,18,25]$. Trotzdem gehen nahezu zwei Drittel von ihnen davon aus, genug für die eigene Gesundheit zu tun.

Nach Lechner et al. [13] kann für eine Diskrepanz zwischen Wahrnehmung und Realität des GVh u.a. mangelndes Wissen oder eine geringere Fähigkeit der Wissensübertragung auf die eigene Person ausschlaggebend sein. Dies sollte bei der Planung von Maßnahmen beachtet werden. Zudem sind die gezeigten Ergebnisse für die ärztliche Betreuung innerhalb und außerhalb des Betriebs bedeutsam, da bei bestimmten gesundheitlichen Risikogruppen (z.B. übergewichtige Raucher) mit einer Fehleinschätzung des GVh zu rechnen ist. Im Gespräch können mögliche Fehleinschätzungen geklärt werden und Betroffene über individuell umsetzbare 
Maßnahmen besonders beraten werden. Die Ergebnisse unserer Untersuchung zeigen aber auch, dass viele verschiedene Faktoren die EGVh beeinflussen. Sie deuten auf ein komplexes Zusammenwirken soziodemographischer und gesundheitsbezogener Faktoren auf die EVGh. Daher sollten zukünftige Forschungsarbeiten das Zusammenspiel der GVh-Faktoren und EGVh detaillierter untersuchen. So sollte neben dem Einfluss und $\mathrm{Zu}$ sammenspiel der untersuchten GVhFaktoren kA, Übergewicht und Rauchen auf die EGVh älterer Beschäftigter u. a. auch der Einfluss weiterer GVhFaktoren, wie Ernährungsgewohnheiten und Alkoholkonsum, in zukünftigen Studien geprüft werden. Auch die Gründe, warum manche Risikogruppen eher zustimmen, genug für die eigene Gesundheit zu tun, sollten in zukünftigen Studien, z. B. mithilfe qualitativer Methoden, spezifischer untersucht werden.

\section{Fazit für die Praxis}

- Die Ergebnisse bestätigen, dass ein deutliches Förderungspotenzial gesunden Verhaltens bei älteren Beschäftigten besteht: Die absolute Mehrheit der Befragten sind übergewichtig bis adipös, bewegen sich nicht ausreichend und etwa ein Drittel raucht. Dennoch gehen zwei Drittel von ihnen davon aus, genug für ihre Gesundheit zu tun.

\section{- Einige Personengruppen schätzten} ihr Verhalten weniger realistisch ein als andere, z. B. übergewichtige Raucher, Nichtaktive mit schlechter körperlicher Gesundheit, Nichtaktive mit geringerer Bildung.

- Dies sollte bei der Gesundheitsberatung sowie bei Angeboten der Gesundheitsförderung und Prävention, innerhalb und außerhalb des Betriebs, berücksichtigt werden.

- Die Gründe, warum diese Gruppen eine weniger realistische Einschätzung haben als andere, sollte in zukünftigen Studien genauer erforscht werden.

\section{Korrespondenzadresse}

Daniela Borchart, M. Sc.

Fachgebiet Arbeitswissenschaft, Bergische Universität Wuppertal

Gaußstraße 20, 42119 Wuppertal, Deutschland borchart@uni-wuppertal.de

Förderung. Die vorliegende Arbeit wurde gefördert durch die BARMER Krankenkasse im Rahmen der Förderung des lidA III-BGF Teilprojekts, Förderkennzeichen: 2016-379.

Funding. Open Access funding provided by Projekt DEAL.

\section{Einhaltung ethischer Richtlinien}

Interessenkonflikt. D. Borchart, H.M. Hasselhorn und J.-B. du Prel geben an, dass kein Interessenkonflikt besteht.

Für diese Studie liegt das Votum der Ethikkommission der Universität Wuppertal vor (5. Dezember 2008). Von allen befragten Studienteilnehmern liegt eine informierte Einverständniserklärung („informed consent ${ }^{\prime \prime}$ ) vor.

\section{Literatur}

1. Atallah N, Adjibade M, Lelong H (2018) How healthy lifestyle factors at midlife relate to healthy aging. Nutrients 10:854

2. Baltes-Götz B (2013) Behandlung fehlender Werte in SPSS und Amos. https://www.uni-trier.de/ fileadmin/urt/doku/bfw/bfw.pdf. Zugegriffen: 13. Nov. 2019

3. Bitzer EM, Sørensen K (2018) Gesundheitskompetenz - Health Literacy. Gesundheitswesen 80:754-766

4. Dare S, Mackay DF, Pell JP (2015) Relationship between smoking and obesity: a cross-sectional study of 499,504 middle-aged adults in the UK general population. PLoS ONE 10(4):e123579

5. Dragano N, Wahl S (2015) Zielgruppenspezifisches Gesundheitsmanagement: Hintergründe, Strategien und Qualitätsstandards. In: Badura B, Ducki A, Schröder H et al (Hrsg) Fehlzeiten-Report 2015. Springer, Berlin/Heidelberg, S21-29

6. Duncan DT, Wolin KY, Scharoun-Lee $M$ et al (2011) Does perception equal reality? Weight misperception in relation to weight-related attitudes and behaviors among overweight and obese US adults. Int J Behav Nutr Phys Act 8:20

7. Finger JD, Mensink GBM, Lange C (2017) Gesundheitsfördernde körperliche Aktivität in der Freizeit bei Erwachsenen in Deutschland. J Health Monit 2(2):37-44

8. Harris KK, Zopey M, Friedman TC (2016) Metabolic effects of smoking cessation. Nat Rev Endocrinol 12(5):299-308

9. Hasselhorn HM, Peter R, Rauch A et al (2014) Cohort profile: the lidA Cohort Study - a German cohort study on work, age, health and work participation. Int J Epidemiol 43(6):1736-1749

10. Jöckel K-H, Babitsch B, Bellach B-M et al (1998) Messung und Quantifizierung soziodemographischer Merkmale in epidemiologischen Studien.
In: Ahrens W, Bellach B-M, Jöckel K-H (Hrsg) Messung soziodemographischer Merkmale in der Epidemiologie. MMV Medizin Verlag, München, S 7-38

11. Kappesser J, Hermann C (2013) Entstehung und Aufrechterhaltung von chronischen Schmerzen. Psychotherapeut 58:503-517

12. Krug S, Jordan S, Mensink G et al (2013) Körperliche Aktivität - Ergebnisse der Studie zur Gesundheit Erwachsener in Deutschland (DEGS1). Bundesgesundheitsbl 56:765-771

13. Lechner L, Bolman C, Van Dijke M (2006) Factors related to misperception of physical activity in The Netherlands andimplicationsforhealth promotion programmes. Health Promot Int 21(2):104-112

14. Lenartz N, Soellner R, Rudinger G (2014) Gesundheitskompetenz: Modellbildung und empirische Modellprüfung einer Schlüsselqualifikation für Gesundes Leben. ZErwachsenenbild 2:29-32

15. Mensink GBM, Schienkiewitz A, Haftenberger M et al (2013) Übergewicht und Adipositas in Deutschland - Ergebnisse der Studie zur Gesundheit Erwachsener in Deutschland (DEGS1). Bundesgesundheitsbl 56:786-794

16. Nübling $M$, Andersen HH, Mühlbacher A (2006) Entwicklung eines Verfahrens zur Berechnung der körperlichen und psychischen Summenskalen auf Basis der SOEP - Version des SF 12 (Algorithmus). DIW, Berlin

17. Pate RR, Pratt M, Blair SN et al (1995) Physical activity and public health. J Am Med Assoc 273:402-407

18. Schienkiewitz A, Mensink GBM, Kuhnert $R$ et al (2017) Übergewicht und Adipositas bei Erwachsenen in Deutschland. J Health Monit 2(2):21-28

19. Schröder H, Kersting A, Gilberg R, Steinwede $J$ (2013) Methodenbericht zur Haupterhebung lidA - leben in der Arbeit. FDZ-Methodenreport 01/2013. Institut für Arbeitsmarkt- und Berufsforschung. http://doku.iab.de/fdz/reporte/2013/ MR_01-13.pdf.Zugegriffen: 13. Nov. 2019

20. Schulz PJ, Hartung U (2016) The future of health literacy. In: Schaeffer D, Pelikan J, KochAlbrecht T(Hrsg) Health Literacy. Die zunehmende Bedeutung von Wissen und Kompetenzen für die Gesundheit. Hogrefe, Bern, S79-92

21. van Sluijs EMF, Griffin SJ, van Poppel MNM (2007) A cross-sectional study of awareness of physical activity:associations with personal, behavioral and psychosocial factors. Int J Behav Nutr Phys Act 4:53

22. Sørensen K, Van den Broucke S, Fullam J et al (2012) Health literacy and public health: a systematic review and integration of definitions and models. Bmc Public Health 12:80

23. Stenholm S, Head J, Kivimäki M (2016) Smoking, physical inactivity and obesity as predictors of healthy and disease-free life expectancy between ages 50 and 75: a multicohort study. Int J Epidemiol 45(4):1260-1270

24. World Health Organization (WHO) (2019) Body mass index - BMI. http://www.euro.who.int. Zugegriffen: 13. Nov. 2019

25. Zeiher J, Kuntz B, Lange C (2017) Rauchen bei Erwachsenen in Deutschland. J Health Monit 2(2):59-65 